\title{
A Project to Install Water-Cherenkov Detectors in the Antarctic Peninsula as part of the LAGO Detection Network
}

Dasso S. ${ }^{a, b, c}$, Gulisano A.M. ${ }^{a, c, d}$, Masías-Meza J.J. ${ }^{c}$ and Asorey H. ${ }^{e}$, for the LAGO Collaboration ${ }^{f}$

a Instituto de Astronomía y Física del Espacio (UBA-CONICET), Buenos Aires, Argentina.

${ }^{b}$ Departamento de Ciencias de la Atmósfera y los Océanos, Facultad de Ciencias Exactas y Naturales, Universidad de Buenos Aires (UBA), Buenos Aires, Argentina.

${ }^{c}$ Departamento de Física and IFIBA, Facultad de Ciencias Exactas y Naturales, Universidad de Buenos Aires (UBA), Buenos Aires, Argentina.

${ }^{d}$ Instituto Antártico Argentino, DNA, Buenos Aires, Argentina.

${ }^{e}$ Laboratorio de Detección de Partículas y Radiación, Instituto Balseiro y Centro Atómico Bariloche, Bariloche, Argentina. Escuela de Física - Universidad Industrial de Santander, Bucaramanga, Colombia.

$f$ lagoproject.org, full list of members and institutions at lagoproject.org/collab.html. E-mail: lago-pi@lagoproject.org

\begin{abstract}
Due to the geomagnetic shielding, particle detectors located at high latitudes allow the observation of cosmic rays (CRs) with lower energies than those located at middle or low latitudes. Thus, Antarctica is a privileged place to study CRs having the lowest energies that can be observed from ground level. The Latin American Giant Observatory (LAGO) consists on a network of waterCherenkov detectors (WCDs) located in nine countries of Latin America, to study with extreme detail the flux of CRs from ground level. The main scientific objectives are oriented to address several problems of astrophysics, space physics and atmospheric physics. In particular, LAGO has started to develop a Space Weather program. A project to install WCDs in the Argentinean Marambio station, located at the Antarctic Peninsula, is being developed as the first antarctic node of LAGO. In this work, we present several aspects of the project, including information about the site, the detector, and studies of geomagnetic and atmospheric properties, that permit us to characterize several useful properties of this location. Results from this new LAGO site will provide important insight for Space Weather, and will provide knowledge to better understand the Sun-Earth coupling.
\end{abstract}

The 34th International Cosmic Ray Conference,

30 July- 6 August, 2015

The Hague, The Netherlands 


\section{Introduction}

One of the aims of the Latin American Giant Observatory (LAGO, previsouly known as Large Aperture Gamma ray burst Observatory, see [1]) is the design, instalation, deployment and operation of an extended astroparticle observatory to a global scale.

The project is operated by the LAGO Collaboration, which is a collaborative, distributed, and not centralized network, composed of researchers and students from nine countries in Latin America: Argentina, Bolivia, Brazil, Colombia, Ecuador, Guatemala, Mexico, Peru and Venezuela. They have recently joined institutions in Spain. The LAGO detection network is formed by particle detectors, individual or in small arrays, located at ground level and installed at different sites. For a details of different LAGO detectors at each LAGO site, see [2]. The network covers a wide latitudinal distribution (currently from Mexico to Patagonia and soon in Antarctica, as will be seen in the development of this paper) and spans a huge range of altitudes (from sea level to over $5000 \mathrm{~m}$ asl), covering an extensive range of geomagnetic rigidities cutoff, and different levels of absorption and reaction at the atmosphere. In addition to the Antartic site that is presented in this paper, other LAGO sites are also starting or will start to operate in LAGO (for more details see $[3,4,5]$ ). The strategy of LAGO to be able to storage, catalogue and preserve its huge amount of data (from both, observations and simulations) can be found in [6].

One the the recent main scientific aims of LAGO is focussed on the study of astroparticles in the context of space weather and atmospheric radiation. Forbush events (see e.g., [7]) are associated with decreases of cosmic rays flux at ground level, due to the shielding in the solar wind, produced by huge interplanetary transient structures, that are recorded by neutron monitors as well as by other ground detectors, as for instance water Cherenkov detectors (WCDs) (e.g., [8]). Occasionally, solar emission of cosmic rays of sufficient energy and intensity as to raise radiation levels at ground level, can be detected by neutron monitors. These events are called Ground Level Enhancements (GLEs). GLEs are of major interest because an enhanced relativistic proton and electron flux can pose a hazard to astronauts, air crews, and also aircraft electronics, then its observation can provide the earliest direct indication of an impending space radiation storm (see e.g., [9]). They can be observed with proper surface detectors located at different appropriate locations of the terrestrial globe, mainly at high latitude regions.

The trajectory of cosmic rays in the far space ambient of the terrestrial environment is mainly affected by the electromagnetic fields that are there. Due to the fact that these particles do not achieve significant effects on the fields (i.e. they are not enough and they have not enough energy as to be significant sources of electric fields or electric currents), they can be treated as passive particles embeded in pre-set fields. In particular, interplanetary conditions or the presence of geomagnetic fields can produce very important effects on these particles, and frequently they produce particle modulation that can be observed using ground particle detectors. When these particles are getting closer to the terrestrial surface, primary cosmic rays interact with the background particles of the atmosphere, producing a huge amount of secondary particles, generated from the interaction between primaries and the atmospheric components. Thus, in order to be able to get a correct interpretation of the signals from the particle detectors, as the LAGO ones, it is necessary to quantify as accurately as possible these effects, in particular those produced by the geomagnetic field and by the atmosphere. 

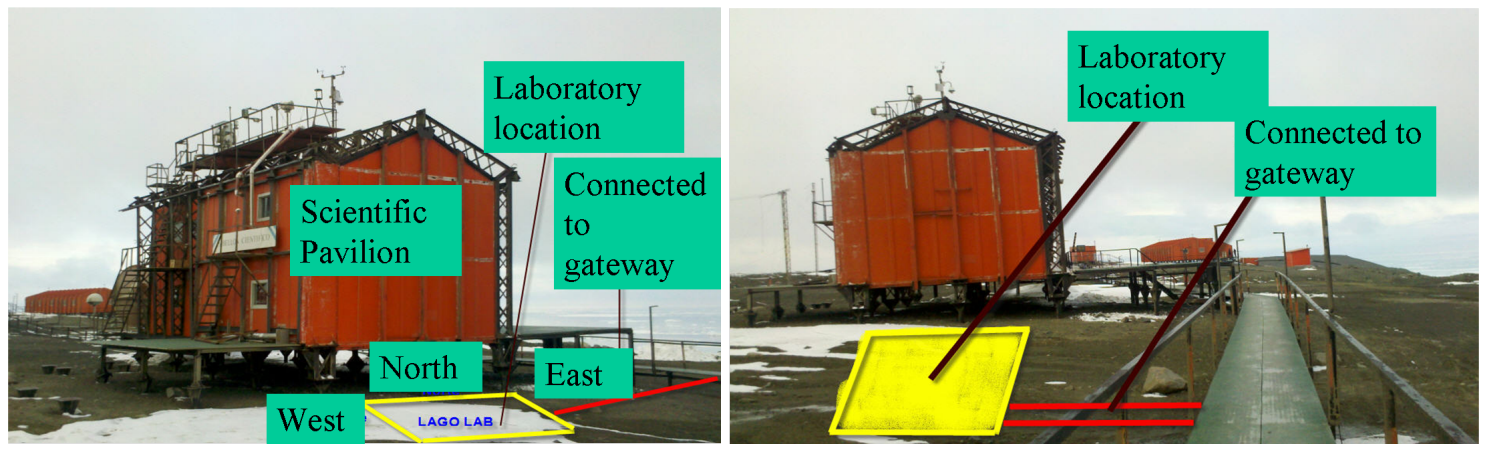

Figure 1: Two views of the LAGO Antarctic facility location.
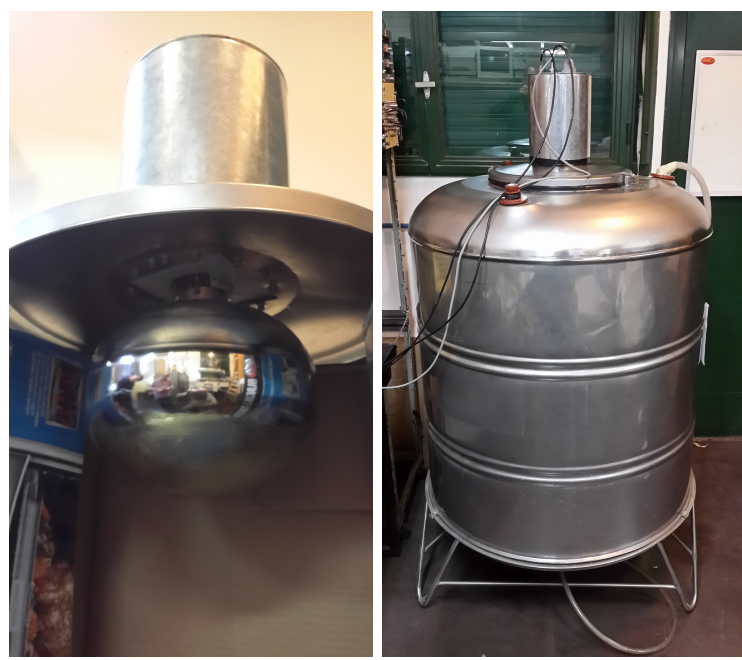

Figure 2: Protopype of the detector.

In Section 2 we describe the place where the detectors will be installed. We then present our studies to characterize the site. In particular, in Section 3 we quantify several effects of the geomagnetic field on primary particles, and in Section 4 we present a comparison of the pressure height profile observed in the local atmosphere with balloons with the typical ones available in cannonical shower simulators. Finally, in Section 5 we present a Summary and our conclusions of the present paper.

\section{The site at Marambio}

The Marambio station of the argentine Antarctic regions is located at Lat. $64^{\circ} 14^{\prime} 24.96$ "S and Long. $56^{\circ} 37^{\prime} 30.34^{\prime \prime} \mathrm{W}$, at 196 meters above sea level. Due to the low temperatures during winter, WCDs at Marambio should be thermally isolated to avoid the uncontrolled freezing of the water and the consequent lost in sensitivity. To do this, we designed facilities where the WCDs will be allocated. The laboratory design allows the installation of up to three WCDs and additional equipment, while in this first stage only two WCDs will be deployed, one detector provided by the Instituto de Astronomía y Física del Espacio (IAFE, UBA-CONICET) and the another one by the Centro Atómico Bariloche (CAB, CNEA). This building takes into account the gateway 


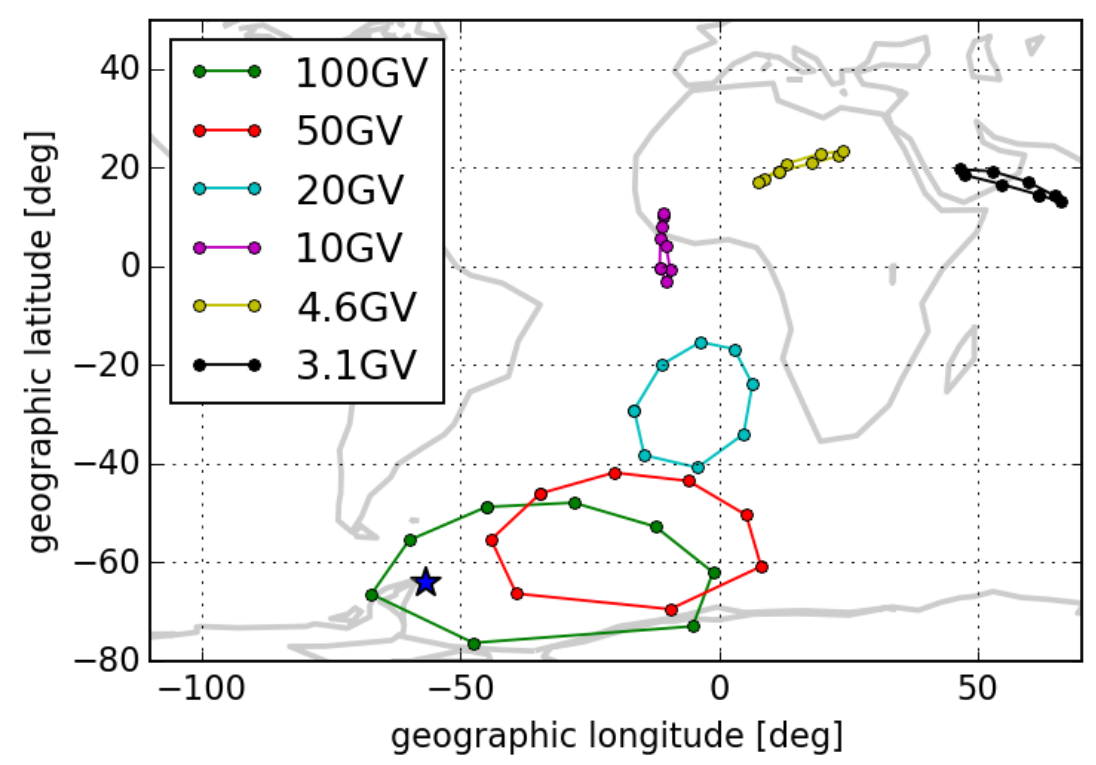

Figure 3: Asymptotic directions of primary protons before interacting with the geomagnetic field, for different energies and different incidence azimuth angles (MAGCOS numerical simulations).

accesibility, it has permafrost characteristics, and optimal construction materials and assembly. The whole structure will be anchored to the permafrost with a depth of at least one meter. The steel base will be elevated $1 \mathrm{~m}$ from ground, to diminish the impact of snow accumulation. The new building will be built next to the current location of the central scientific pavillon of the Marambio base, and will have access by using an external corridor. Figure 1 shows the place where the LAGO building will be constructed and Figure 2 shows a prototype of the detector that was contructed at the Instituto de Astronomía y Física del Espacio, in Buenos Aires. In the left panel of the figure can be noticed that the base of the PMT is isolated above the water container, in order to decrease the level of humidity. Several pieces of the detector has been tested at temperatures well below zero Celsius, to ensure its quality after suffering any unforeseen situation with low temperatures regimes in the Antarctic soil.

The analysis of the data from this WCD at Antartic will allow us to make studies of the background cosmic rays rate, similar to the ones that LAGO is currently making in different sites (e.g., at Chacaltaya [10]), but with higher counting rates and with the impact from primary particles having lower energies.

\section{Geomagnetic field effects: Numerical Simulations}

In this section we present results of numerical simulations of the trajectory of charged particles arriving to Marambio site, using the MAGCOS code (http://cosray.unibe.ch/laurent/magnetocosmics). For details of the simulations developed see [11]. Similar studies as the ones presented here for Marambio, but for another LAGO sites can be found in [12].

Figure 3 shows the asymptotic directions of protons arriving at the LAGO Marambio site (projected on the Earth's surface) for $15^{\circ}$ zenith incidence and eight equispaced incidence azimuth 


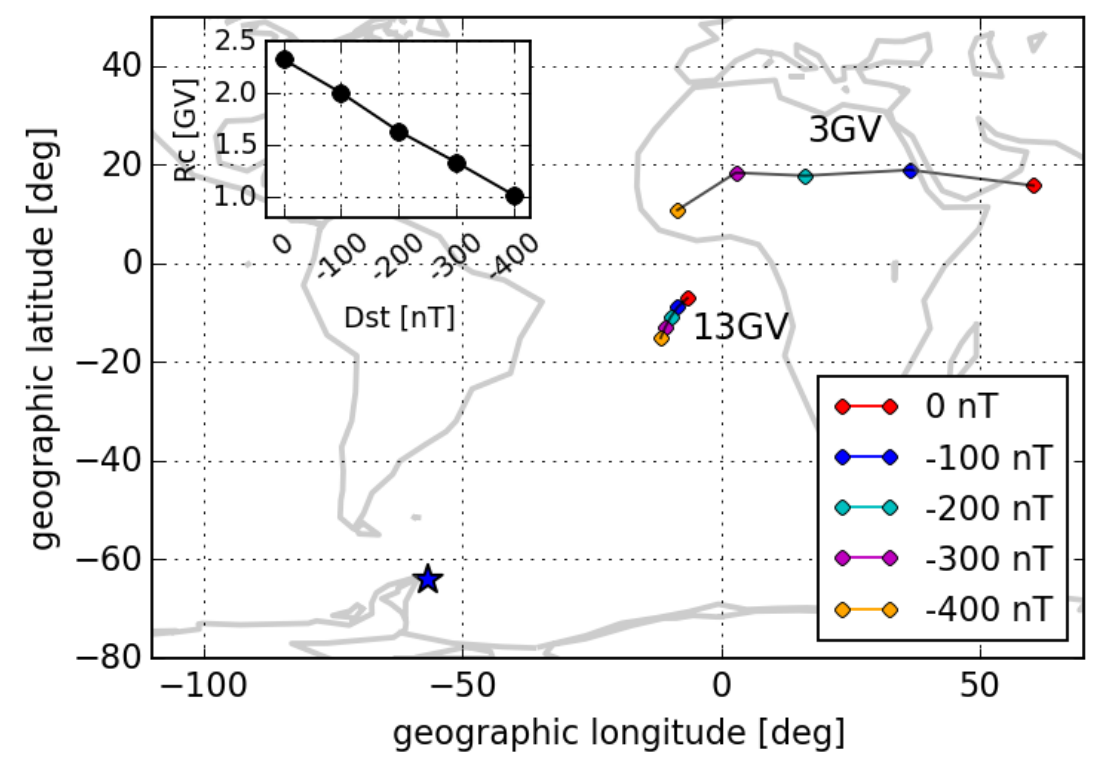

Figure 4: Asymptotic directions for vertical incidence, covering different levels of geomagnetic activity. The inset shows the rigidity cutoff for different geomagnetic conditions, according to the Dst index.

values $\left(45^{\circ}, 90^{\circ}, 135^{\circ}, 180^{\circ}, 225^{\circ}, 270^{\circ}, 315^{\circ}\right.$, and $\left.360^{\circ}\right)$. For this calculation we use the International Geomagnetic Reference Field (IGRF10) for modeling the Earth magnetic field. Figure shows these asymptotic directions for rigidities 3.1, 4.6, 10, 20, 50 and $100 \mathrm{GV}$. We can see that as the particle rigidity decreases, the asymptotic trajectories get closer to the equatorial region. From a detailed analysis of the simulations done it is possible to conclude that these particles are mostly deflected at heights lower than $\sim 2 \mathrm{RE}$, where the configuration of the geomagnetic field is strongly dominated by a dipolar component.

However, during periods of geomagnetic storms, the non-dipolar component of Bgeo (produced mainly by magnetospheric electric currents) can significantly affect the trajectory of these particles. We develope simulations to compute asymptotic directions for different geomagnetic conditions using the Dst index as the proxy and considering the Tsyganenko 2001 (TSY01) model [13]. In figure 4 we show the asymptotic directions of particles with vertical incidence and rigidities for different values of $D s t$ ( $D s t=0,-100,-200,-300$ and $-400 \mathrm{nT}$ ). We note the shift in longitude for these asymptotic directions, to eastward for higher geomagnetic activity. The inset of 4 also shows the rigidity cutoff for different $D s t$ values, getting $R c$ as low as $\sim 1 \mathrm{GV}$ for $D s t \sim-400 \mathrm{nT}$. We find that the rate of variation of $R c$ with $D s t$ is $\sim-0.003 \mathrm{GV} / \mathrm{nT}$ at Marambio.

\section{Study of the Atmosphere at Marambio}

Numerical simulations of cosmic particle cascades in the terrestrial atmosphere require a detailed knowledge of the pressure height profile in the site. Some numerical models (e.g. CORSIKA code) need specific information about the height profiles of the simulated site. Ten different MODTRAN models are commonly used in CORSIKA simulations. 

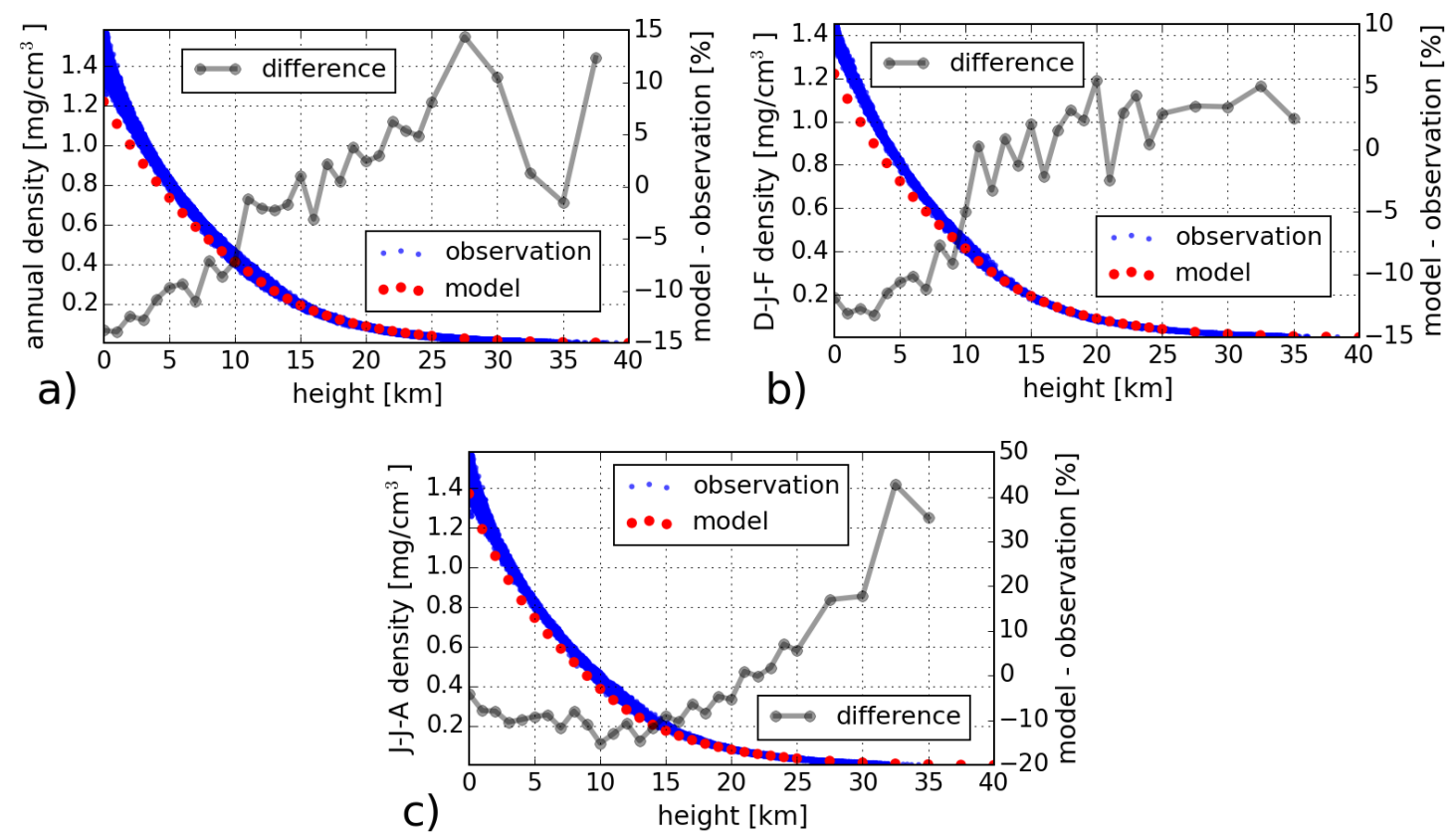

Figure 5: A comparison of atmospheric density models from MODTRAN with the observed with baloons at Marambio base. The atmospheric MODTRAN models compared here are: a) the standard one, b) the subartic summer, and c) the subartic winter.

Presently, a typical CORSIKA shower simulation at the LAGO Marambio site can be done assuming one of the available atmospheric MODTRAN models. In order to accomplish a detailed depiction for the profile at this site, in this section we analyze balloon soundings data measured at 12 UTC by the argentine National Weather Service (Servicio Meteorológico Nacional, SMN) from 1998 until 2014, at Marambio. We computed the density height profile based on the height pressure and temperature profiles observed from the soundings as follows: we take into account the typical atmospheric composition, their molecular masses and the Dalton law for partial pressures for ideal gases. Thus, from the pressure and temperature data for each altitude, we compute the density height profile.

A comparison between the characterization that we obtain and the ones frequently used in simulations performed with CORSIKA, is presented in Figure 5. In particular, we compare our results with three pre-defined atmospheric (MODTRAN) models of CORSIKA: a) 'Standard', b) 'Sub-Artic Summer', and c) 'Sub-Artic Winter'. We make the comparisons using appropriate different seasons. We find that the profile for the 'Sub-Artic Winter' model deviates significantly from the local profile observed (between $10 \%$ and $40 \%$ for altitudes larger than $25 \mathrm{~km}$ ). However, the deviation of the density profiles from the 'Standard' and 'Sub-Artic Summer' models, respect to the ones observed with the baloons of SMN at Marambio, is not so high. However, the found differences can affect a proper simulation. In particular it differs in less than $\sim 10 \%$ for the 'Standard' and even less (less than that 5\%) for the 'Sub-Artic Summer' model. We notice that the larger differences correspond to larger altitudes. And in general the models lightly underestimate the observations at lower altitudes (lower than $\sim 10 \mathrm{~km}$ ) and make significantly overestimations at 
altitudes higher than $\sim 15 \mathrm{~km}$, where details of the shower simulations are crucial. The presented results can help to take the decision of what pre-set model to use, but also to construct a new one for Marambio, based on local observations. So that, they will improve the quality of numerical simulation for cosmic rays showers at the atmosphere at Marambio.

\section{Summary and Conclusions}

In this work we presented the project of the LAGO collaboration for installing WCDs in the argentine Antarctica. We also presented some studies for this site that will allow a better interpretation of the acquired data. Our results quantify asymptotic directions of primaries for different rigidities, and also rigidities cutoff for different geomagnetic conditions. We presented a study of the typical atmospheric conditions for this site using baloons observations, and compared them with the MODTRAN models commonly used in CORSIKA, quantifying relative differences. We conclude that the 'Sub-Artic Summer' model is the most appropriated one at Marambio for the months December, January and February. The results we found here will improve the development of showers numerical simulations we plan to do in the near future in the Antarctic node of the LAGO collaboration, getting a better simulation of these secondary showers. In particular, we plan to develope showers simulations using new models based on these balloon observations.

It is a unique moment for making combined studies of astroparticles flows, combining onboard spacecraft detectors (for nucleons with energies between a few tens of $\mathrm{keV}$ to $100 \mathrm{MeV}$ per nucleon, e.g., instruments aboard the ACE, SOHO and SAMPEX probes) with ground particle detectors (covering energies from $\sim 1 \mathrm{GeV}$ up to $\sim 1 \mathrm{TeV}$, e.g., neutron monitors, muon telescopes and WCDs from the LAGO and Auger observatories).

While neutron monitors are proportional counters that measure the total number of particles (without energy discrimination), the modern WCDs that will be installed at Antarctic are able to measure the histogram of the energy deposited by the secondary particles observing only at a single geographic localization. These histograms can be re-interpreted in function of the energy of the primary cosmic rays from numerical simulations, and thus for instance will be possible to measure for the first time the effects of the passage of a magnetic cloud on the energy spectrum of galactic cosmic rays at the terrestrial environment.

The new site of LAGO in Antartic will be able to observe two particular classes of physical events (Forbush drecreases and GLEs), which are of major interest in space weather.

These combined studies are complementary and will make it possible to achieve a deeper understanding of the space environment. They will contribute to a better comprehension of different effects of the Sun-Earth coupling on the cosmic radiation levels at different levels of the terrestrial environment and also at the ground level of Earth.

\section{Acknowledges}

This work was partially supported by the Argentinean grants PICT-2013-1462 (FONCyTANPCyT), UBACyT 20020120100220 (UBA), and PIP-11220130100439CO (CONICET). We acknowledge SMN for providing baloons data for Marambio, and also to Viviana Lopez for helping 
us to understand its format. The LAGO Collaboration thanks the Pierre Auger Collaboration for its continuous support.

\section{References}

[1] H. Asorey for the LAGO Collaboration, "LAGO: the latin american giant observatory," in this Proc., vol. in press, 2015.

[2] I. Sidelnik for the LAGO Collaboration, "The sites of the latin american giant observatory," in this Proc., vol. in press, 2015.

[3] A. Galindo and others for the LAGO Collaboration, "Sensitivity of lago and calibration of a water-cherenkov detector in sierra negra, méxico.," in this Proc., vol. in press, 2015.

[4] L. Otiniano and others for the LAGO Collaboration, "Development of a high altitude lago site in peru," in this Proc., vol. in press, 2015.

[5] S. Vargas and others for the LAGO Collaboration, "LAGO ecuador, implementing a set of wcd detectors for space weather research: first results and further developments," in this Proc., vol. in press, 2015.

[6] H. Asorey and others for the LAGO Collaboration, "Data accessibility, reproducibility and trustworthiness with lago data repositories," in this Proc., vol. in press, 2015.

[7] G. Wibberenz, L. R. J.A., M. Potgieter, and J. Bieber, Transient effects and disturbed conditions: observations and simple models, vol. 83. 1998.

[8] The Pierre Auger Collaboration, "The Pierre Auger Observatory scaler mode for the study of solar activity modulation of galactic cosmic rays,” JINST, vol. 6, p. P01003, 2011.

[9] J. W. Bieber, J. Clem, P. Evenson, R. Pyle, A. Sáiz, and D. Ruffolo, "Giant Ground Level Enhancement of Relativistic Solar Protons on 2005 January 20. I. Spaceship Earth Observations," AstroPhysical J., vol. 771, p. 92, July 2013.

[10] C. Sarmiento-Cano and others for the LAGO Collaboration, "Analysis of background cosmic ray rate in the 2010-2012 period from the LAGO detectors at chacaltaya," in this Proc., vol. in press, 2015.

[11] J. Masias-Meza and S. Dasso, "Geomagnetic effects on cosmic ray propagation under different conditions for Buenos Aires and Marambio, Argentina," Sun and Geosphere, vol. 9, pp. 41-47, Jan. 2014.

[12] M. Suarez and others for the LAGO Collaboration, "The LAGO space weather program: Directional geomagnetic effects, background fluence calculations and multi-spectral data analysis," in this Proc., vol. in press, 2015.

[13] N. A. Tsyganenko, "A model of the near magnetosphere with a dawn-dusk asymmetry 1. Mathematical structure," Journal of Geophysical Research (Space Physics), vol. 107, p. 1179, Aug. 2002. 